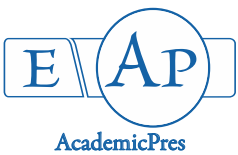

Rhetso T et al. (2021)

Notulae Scientia Biologicae

Volume 13, Issue 2, Article number 10791

DOI: $10.15835 / \mathrm{nsb} 13210791$

Research Article

\title{
GC-MS based metabolite profiling and antioxidant activity of solvent extracts of Allium chinense G Don leaves
}

\section{Thejanuo RHETSO, Roopa M. SESHADRI, Shubharani RAMNATH, Sivaram VENKATARAMEGOWDA*}

Bangalore University, Department of Botany, Jnana Bharathi Campus, Bengaluru-560056, India; thejanuo.rhetso@gmail.com; roopams.iyengar@gmail.com; shubharani.bolar19@gmail.com; sivaram900@gmail.com ("corresponding author)

\begin{abstract}
Allium chinense, a main source of "Xiebai" drug in Chinese traditional medicine and commonly known as Ganoderma lucidum belongs to the family Amaryllidaceae. The main focus of this research was to quantify the secondary metabolites, antioxidant potential and study the GC- MS based metabolite profile of different solvent leaf extracts of $A$. chinense. The reports on the bioactive compounds of $A$. chinense leaves are still insufficient compared to the bulb; hence this study was carried out to understand the bioactive compounds present in $A$. chinense leaves using different solvents of varying polarity. Our investigation showed that the ethanol extract contained the highest saponin, flavonoid, phenol, and DPPH scavenging activity. Further, metabolite profiling revealed a total of forty-eight compounds, indicating a diverse range of phytochemicals present in the four extracts. The highest number of compounds were observed in ethanol extract (15) followed by chloroform extract (13), petroleum ether extract (11) and methanol extract (9). Some of the major compounds identified in the four solvents are octacosane (27.11\%), heptadecane (19.66\%), eicosane (18.51\%), ethyl palmitate (18.50\%), phytol (17.68\%) and phytol acetate (17.30\%). In conclusion, this study highlights that $A$. chinense leaf extracts contain high saponins, terpenes and alkanes which could be a potential source of a new beneficial drug.
\end{abstract}

Keywords: Allium chinense, bioactive compounds; metabolite profile; scavenging activity

\section{Introduction}

Medicinal herbs are a rich source of a bioactive compound and considered to play a beneficial role in traditional and modern health care delivery systems. The bioactive compounds synthesized from these plants provide the raw material for the cosmetic and pharmaceutical industries (Kretovich, 2005). Allium chinense $\mathrm{G}$ Don, is a medicinal herb from the Amaryllidaceae family, commonly known as the oriental onion or Ganoderma lucidum in vegetables. The plant is widely distributed in India's North-East states and grows well in 15-30 ${ }^{\circ} \mathrm{C}$ temperature with moderate fertile soils (Lim et al., 2015). It has a strong onion-like odor and known for its rich organo-sulfur (Pino et al., 2001; Liu et al., 2014) and saponin (Sobolewska et al., 2020) content and is also the original source of the famous Chinese medicine "Xiebai", as it functions like a tonic to the digestive system (Yao et al., 2016). Saponins from edible plants have been reported to have diverse biological 
functions, including antitumor effects (Wang et al., 2019). The essential oil of $A$. chinense bulb and leaf has been reported to have sulfide containing compounds (Pino et al. 2001; Liu et al., 2014) which are the main components for odour and it is effective in breaking seed and bud dormancy (Hosoki et al., 1986; Kubota et al., 2000). However, there is no report on quantification, GC-MS based metabolite profiling, and antioxidant potential of different solvents extracts of $A$. chinense leaves. The investigation on bioactive compounds of $A$. chinense leaves is still inadequate compared to the research findings on the bulb. Hence, the present study aimed to identify and characterize biomolecules by GC-MS analysis and to determine the antioxidant potential of $A$. chinense leaf in different solvent extracts.

\section{Materials and Methods}

\section{Plant material collection and preparation of extracts}

A. chinense plants were collected from agriculture fields in Viswema village $\left(25.5615^{\circ} \mathrm{N}, 94.1450{ }^{\circ} \mathrm{E}\right)$, Kohima, Nagaland, India, and was identified and authenticated by Dr. V. Rama Rao, Regional Ayurvedic Research Institute for Metabolic Disorders (Central Council for Research in Ayurvedic Sciences, Ministry of AYUSH, Govt. of India) Bengaluru-560109. The voucher specimen RRCBI-mus 244 was preserved and deposited in Botany Department, Bangalore University, Bangalore. The leaves were carefully washed with distilled water and shade-dried till all the moisture contents were removed. The dried leaves were ground using a mixer to a fine powder and extracted using Soxhlet extractor (Merculieff et al., 2014) with different solvents viz, ethanol, methanol, chloroform, and petroleum ether. The extracts were concentrated using a rotary evaporator at $30-40^{\circ} \mathrm{C}$ for petroleum ether and chloroform and $40-50^{\circ} \mathrm{C}$ for methanol and ethanol stored in a bottle in a cool $\left(4^{\circ} \mathrm{C}\right)$, dark environment for further use.

\section{Quantitative analysis}

Determination of total phenols

The total phenols in different solvent extracts of $A$. chinense leaf were evaluated using FC (FolinCiocalteu) spectrophotometric method (Khan and Bhat, 2018). The standard curve was plotted using gallic acid at $1-100 \mu \mathrm{g} / \mathrm{ml}$ concentrations. Plant extracts at $1 \mathrm{mg} / \mathrm{ml}$ were mixed with $5 \mathrm{ml}$ of FC (1:10) and $4 \mathrm{ml}$ of sodium bicarbonate $(7.5 \%)$ and the mixture was incubated for half an hour in the dark at $20{ }^{\circ} \mathrm{C}$ to allow the reaction to take place. The absorbance was measured at a wavelength of $765 \mathrm{~nm}$ against blank.

\section{Estimation of total alkaloids}

The total alkaloids were quantified using atropine as a reference standard at 200-1000 $\mu \mathrm{g} / \mathrm{ml}$ concentration to obtain the calibration curve (Tan, 2018). The extracts $(1 \mathrm{mg} / \mathrm{ml}$ ) were mixed with $2 \mathrm{ml}$ of $2 \mathrm{~N} \mathrm{HCl}$ and washed with $5 \mathrm{ml}$ of chloroform. The solution was vortexed and layers were separated using a micropipette. Later, the separated layer was taken in a test-tube and $5 \mathrm{ml}$ of BCG (Bromocresol Green) solution and phosphate buffer ( $\mathrm{pH} 4.7$ ) was added and vortexed. A yellow color complex observed at the bottom was carefully removed by using pipette and absorbance was measured at a wavelength of $470 \mathrm{~nm}$.

\section{Estimation of total flavonoids}

The quantification of total flavonoids content in different solvent extracts was determined using the $\mathrm{AlCl}_{3}$ (Aluminum trichloride) method. Catechin was used as a standard (Aryal et al., 2019) at 20-100 $\mu \mathrm{g} / \mathrm{ml}$ concentration to plot the standard calibration graph. Plant extracts $(0.5 \mathrm{ml})$ with $1 \mathrm{mg} / \mathrm{ml}$ concentration was suspended in distilled water $(2 \mathrm{ml})$ in a test tube, $0.15 \mathrm{ml}$ of $5 \% \mathrm{NaNO}_{2}$ was added to it and incubated for 6 minutes. Thereafter, $0.15 \mathrm{ml}$ of $10 \% \mathrm{AlCl}_{3}$ was added and incubated again for 6 minutes. Later, $2 \mathrm{ml}$ of $10 \%$ $\mathrm{NaOH}$ was added to it and the final volume was made up to $5 \mathrm{ml}$ using distilled water. The solution was then incubated at room temperature for 15 minutes and the absorbance of the solution was read at $510 \mathrm{~nm}$. 


\section{Estimation of saponins}

The saponin content of the extracts was estimated using the method described by Le et al. (2018). The Plant extract at $1 \mathrm{mg} / \mathrm{ml}$ concentration was taken in a test tube and $500 \mu \mathrm{L}$ of $8 \%$ vanillin and $72 \%$ sulphuric acid was added and incubated for 10 minutes at $60{ }^{\circ} \mathrm{C}$ in a water bath. After incubation, it was allowed to cool to room temperature and absorbance was measured at $544 \mathrm{~nm}$. Quillaia $(200-1000 \mu \mathrm{g} / \mathrm{ml})$ was used as a standard to generate the standard calibration curve.

\section{DPPH free radical scavenging assay}

A. chinense leafs ability in different solvent extracts to scavenge free radicals was evaluated (Vasundhara et al., 2017) using ascorbic acid as the standard and all the tests were performed in triplicate. One $\mathrm{ml}$ of varying plant extract and ascorbic acid with $1-100 \mu \mathrm{g} / \mathrm{ml}$ concentrations were mixed with $3.0 \mathrm{ml} \mathrm{of} \mathrm{DPPH}$ $(0.06 \mathrm{mM})$ and incubated in dark for 15 minutes at room temperature for reaction to occur. The absorbance was measured at a wavelength of $517 \mathrm{~nm}$ using a UV-Vis spectrophotometer. The percentage of inhibition of the extracts and ascorbic acid was calculated using the given formula:

DPPH radical scavenging activity $(\%)=\left[\left(A_{1}-A_{2}\right) / A_{1}\right] \times 100$

Where $A_{1}$ - Absorbance of control (DPPH), $A_{2}$ - Absorbance of extracts

The calibration curve was generated and $50 \%$ inhibition concentration $\left(\mathrm{IC}_{50}\right)$ values were calculated.

\section{$G C-M S$ analysis and identification of compounds}

To obtain the complete chemical profile of $A$. chinense leaf, GC-MS analysis was performed using SHIMADZU QP2010S GC-MS system. The operating condition was set up as follows: and oven temperature was programmed at $70.0^{\circ} \mathrm{C}$; the ion source temperature was set at $200.00^{\circ} \mathrm{C}$ sample was injection mode was split less and sampling time 2.00 min equipped with Rxi-5Sil MS column; length: 30 meters, the carrier gas was helium (99.99\%) at a flow of $1.00 \mathrm{~mL} / \mathrm{min}$; start time $7.00 \mathrm{~min}$; End time $35.75 \mathrm{~min}$; event time $0.50 \mathrm{sec}$; scan range $50-500 \mathrm{~m} / \mathrm{z}$. The chemical compounds were identified based on the peaks observed at different mass-tocharge ratios. Further identification was made by comparing with the standard spectrum existing in the database mass spectral library of the National Institute of Standards and Technology NIST-11 and WILEY 8 library.

\section{Statistical analysis}

The data were analysed using a one-way method of Analysis of variance (ANOVA) at a 5\% probability (P,0.005) by using Prism V. 5.00 (Graphpad Inc. USA). The total phenol, alkaloid, flavonoids and saponin content was estimated using the linear regression equation obtained from the standard graph. All the analysis was carried out in triplicates and expressed as mean \pm SE.

\section{Results and Discussion}

\section{Quantitative analysis}

In recent years, plant metabolites have played an important role in alleviating several ailments and multiple health benefits, the knowledge on phyto components is important to obtain active principles for its highest pharmacological significance. The different solvent extract has a significant difference in the concentration of total phenols, alkaloid, flavonoid, and saponin (Table 1). 
Table 1. Quantitative analysis of phenol, alkaloid, flavonoid, and saponin content in $A$. chinense leaf extracts

\begin{tabular}{|c|c|c|c|c|c|}
\hline $\begin{array}{c}\mathrm{S} \\
\text { No. }\end{array}$ & Sample & Alkaloids $(\mathrm{mg} / \mathrm{g})$ & Flavonoids $(\mathrm{mg} / \mathrm{g})$ & Phenol $(\mathrm{mg} / \mathrm{g})$ & Saponin $(\mathrm{mg} / \mathrm{g})$ \\
\hline & $\begin{array}{c}\text { Linear } \\
\text { regresion } \\
\text { equation for } \\
\text { standard }\end{array}$ & $\begin{array}{c}\mathrm{Y}=0.0003 \mathrm{X}+0.0009 \\
\mathrm{R}^{2}=0.9996\end{array}$ & $\begin{array}{c}\mathrm{Y}=0.0029 \mathrm{X}+0.0031 \\
\mathrm{R}^{2}=0.9992 \\
\text { (Atropine) }\end{array}$ & $\begin{array}{c}\mathrm{Y}=0.0127 \mathrm{X}+0.0252 \\
\mathrm{R}^{2}=0.9991 \\
\text { (Guercetin) }\end{array}$ & $\begin{array}{c}\mathrm{Y}=0.0002 \mathrm{x}+0.003 \\
\mathrm{R}^{2}=0.999\end{array}$ \\
\hline 1 & Ethanol & $49.080 \pm 0.481$ & $38.241 \pm 0.398$ & $35.575 \pm 0.227$ & $515.000 \pm 0.578$ \\
\hline 2 & Chloroform & $200.750 \pm 0.254$ & $12.034 \pm 0.783$ & $22.859 \pm 0.205$ & $322.500 \pm 0.289$ \\
\hline 3 & $\begin{array}{c}\text { Petroleum } \\
\text { ether }\end{array}$ & $184.920 \pm 0.918$ & $15.483 \pm 0.199$ & $17.819 \pm 0.068$ & $351.250 \pm 0.144$ \\
\hline 4 & Methanol & $64.500 \pm 0.770$ & $20.138 \pm 0.542$ & $25.260 \pm 0.273$ & $496.250 \pm 0.722$ \\
\hline
\end{tabular}

The highest concentration of saponins was recorded in ethanol extract, followed by methanol extract, petroleum ether extract and chloroform extract. Quantitative analysis in leaf extract showed a moderately high concentration of alkaloid in chloroform and petroleum ether extract; however, in methanol and ethanol the concentration was moderate. In quantitative estimation, low phenol and flavonoid content was observed in leaf extract; ethanol extract showed higher phenolic and flavonoid content than the other extracts.

Saponin are widely distributed in monocotyledonous families (Sobolewska et al., 2020), and several studies have confirmed that they have an extensive range of pharmacological activities. Steroidal saponins vernoniamyoside $\mathrm{A}, \mathrm{B}$, and $\mathrm{B}_{2}$, possess cytotoxicity activity against BT-549 (Wang et al., 2018), steroidal sapogenin 25 - $R$-spirosta-3, 5-dien-12 $\beta$-ol showed cytotoxicity on $5-8 \mathrm{~F}$ cells a human nasopharyngeal carcinoma cell line (Chu et al., 2018). Saponin subsides coronary heart disease (Yang et al., 2018) and acts as cytotoxic towards the human glioblastoma U87MG and U251 cell lines (Liu et al., 2018). Saponins like diosgenin have great value in the pharma industry and used as substrates in drug and steroid hormone production (Sobolewska et al., 2020). The presence of a high saponin amount might have contributed to the significant use of this plant in traditional medicine and drug discovery. Quantitative analysis of leaf extract showed a moderately high concentration of alkaloid. Plant alkaloids possess anti-inflammatory, anti-depressive, antioxidant, anti-convulsing, anti-amyloid efficacy, antiviral, antifungal, anticancer and antibacterial activity (Hussain et al., 2018; Thawabteh et al., 2019). The analysis showed a moderate concentration of phenol and flavonoid content in the leaf extract. Phenols possess high antioxidant activity (Safari and Ahmady-Asbchin, 2019) and contribute hydrogen and react with nitrogen and reactive oxygen compounds acting as an antioxidant (Pereira et al., 2009). Medicinal plants have a copious amount of flavonoids and are considered potential nutraceuticals, They also regulate numerous pathways for diseases like diabetes, neuro-disease, cancers, and other transmittable diseases (Qiu et al., 2018).

\section{Antioxidant assay}

The free radical scavenging activity was determined in all the four solvent extracts of $A$. chinense leaf viz ethanol, methanol, chloroform, and petroleum ether by the DPPH method. Scavenging activity against free radicals from leaf extracts is shown in (Figure 1, Table 2). 


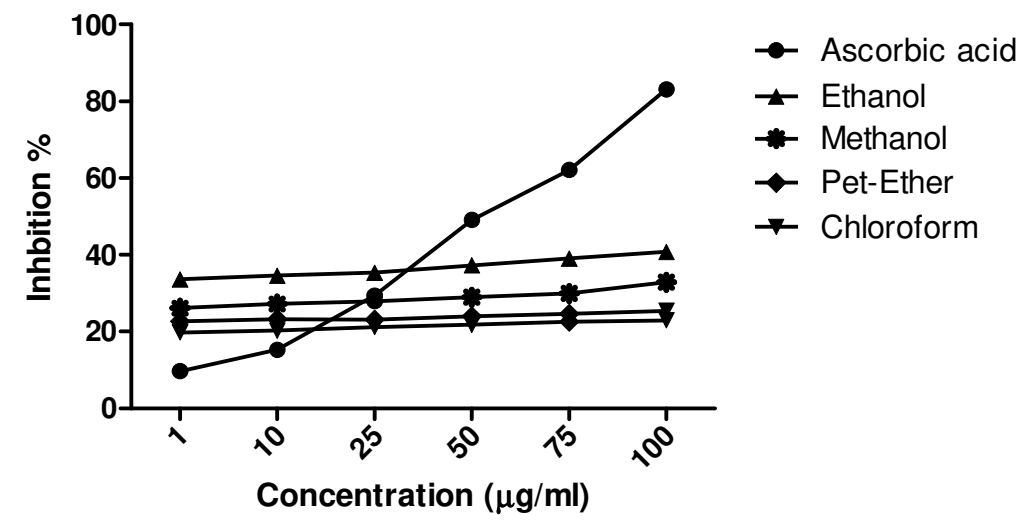

Figure 1. Scavenging activity of ascorbic acid, ethanol, methanol, petroleum ether, and chloroform extract of $A$. chinense leaf

Table 2. Antioxidant activity measured by DPPH scavenging method

\begin{tabular}{|l|c|c|c|c|c|}
\hline $\begin{array}{c}\text { Conc. } \\
(\mu \mathrm{g} / \mathrm{ml})\end{array}$ & Ascorbic acid & Ethanol & Chloroform & Petroleum ether & Methanol \\
\hline 1 & $9.720 \pm 0.498$ & $33.586 \pm 0.141$ & $22.747 \pm 0.162$ & $19.769 \pm 0.289$ & $26.091 \pm 0.576$ \\
\hline 10 & $15.324 \pm 0.382$ & $34.637 \pm 0.240$ & $23.257 \pm 0.180$ & $20.329 \pm 0.232$ & $27.167 \pm 0.622$ \\
\hline 25 & $29.411 \pm 0.214$ & $35.340 \pm 0.184$ & $23.086 \pm 0.238$ & $21.247 \pm 0.140$ & $27.928 \pm 0.042$ \\
\hline 50 & $49.132 \pm 0.526$ & $37.253 \pm 0.139$ & $23.963 \pm 0.299$ & $21.781 \pm 0.468$ & $28.990 \pm 0.083$ \\
\hline 75 & $62.159 \pm 0.574$ & $39.012 \pm 0.281$ & $24.623 \pm 0.099$ & $22.596 \pm 0.554$ & $29.954 \pm 0.591$ \\
\hline 100 & $83.157 \pm 0.417$ & $40.833 \pm 0.412$ & $25.432 \pm 0.585$ & $22.969 \pm 0.587$ & $32.915 \pm 0.582$ \\
\hline $\mathrm{IC}_{50}$ & 55.096 & 228.156 & 1045.287 & 947.025 & 391.529 \\
\hline
\end{tabular}

The $A$. chinense leaf extracts showed moderate scavenging activity in all the four solvents compared to standard ascorbic acid $\left(\mathrm{IC}_{50}=55.096 \mathrm{\mu g} / \mathrm{ml}\right)$ with a significant difference $(\mathrm{p}<0.05)$. Ethanol extract with $\mathrm{IC}_{50}$ value of $228.156 \mu \mathrm{g} / \mathrm{ml}$ was found to exhibit the highest free radical scavenging activity when compared to other solvent extracts, followed by methanol with $\mathrm{IC}_{50}$ value of $391.529 \mu \mathrm{g} / \mathrm{ml}$, petroleum ether with $\mathrm{IC}_{50}$ of $947.025 \mu \mathrm{g} / \mathrm{ml}$, and chloroform extract with IC $_{50}$ value of $1045.287 \mu \mathrm{g} / \mathrm{ml}$.

Free radicals are unstable molecules that cause oxidative stress, triggering cell damage, antioxidants are substances that may prevent or delay cell deterioration, vegetables and fruits are a rich source of antioxidants (Lobo et al., 2010). The percentage of free radical scavenging activity was found to be significantly low, which may be due to a lower concentration of phenol (Wang et al., 2018) and flavonoids in the present study (Liu et al., 2018). Lin et al. (2016) observed mild antioxidant activity in the essential oil of $A$. chinense bulb which supports the present findings.

\section{GC-MS analysis}

Identifying plant's chemical constituents is important for finding new therapeutic agents and GC-MS is the key technology for secondary metabolites profiling in plants. Bioactive compounds identified by GC-MS analysis showed that the leaf extracts have a complex combination of numerous compounds; some of which were present in trace quantities. The GC-MS analysis of methanol, ethanol, chloroform, and petroleum ether extracts of $A$. chinense leaf revealed a total of 48 peaks. The highest number of peaks was observed in ethanol extract. Fifteen compounds were identified in ethanol extract with a run time of $50 \mathrm{~min}$. (Figure 2, Table 3). In the chloroform extract, thirteen peaks were observed with a run time of $45 \mathrm{~min}$. (Figure 3, Table 4). The petroleum ether extract was run for $49 \mathrm{~min}$. (Figure 4, Table 5) where eleven peaks were recorded. A total of 
nine peaks were detected in the chromatogram of methanol leaf extract with a run time of $49 \mathrm{~min}$. (Figure 5 , Table 6). The peaks indicated the number of compounds and the active compounds was confirmed based on retention time, molecular weight, molecular formula, and molecular structure. Among the various phytochemicals identified, phytol and phytol acetate were the most common chemical compounds found in all four extracts.

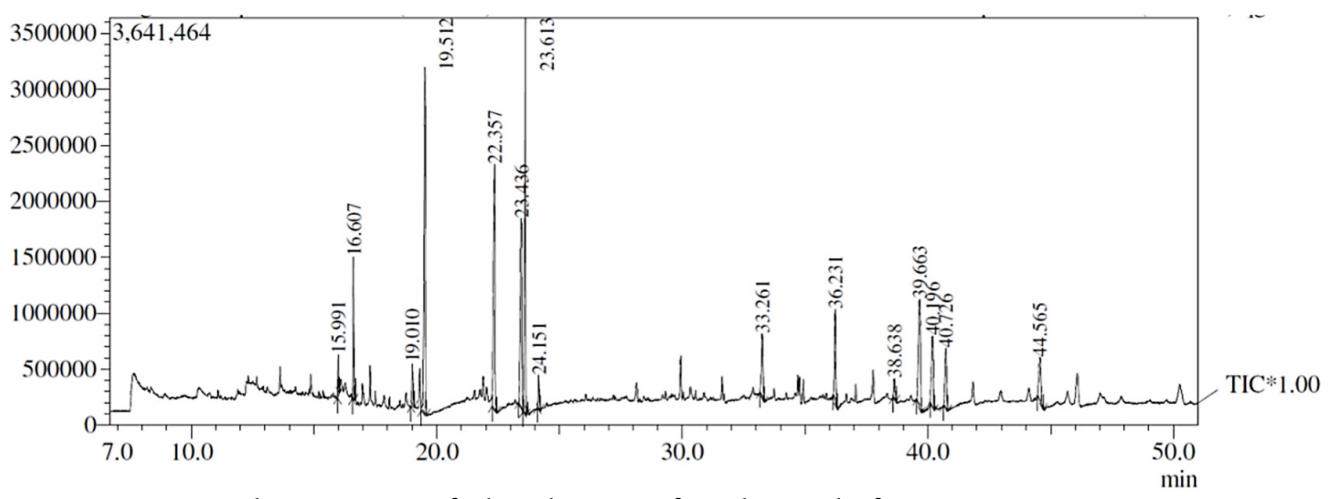

Figure 2. GC-MS chromatogram of ethanol extract of $A$. chinense leaf

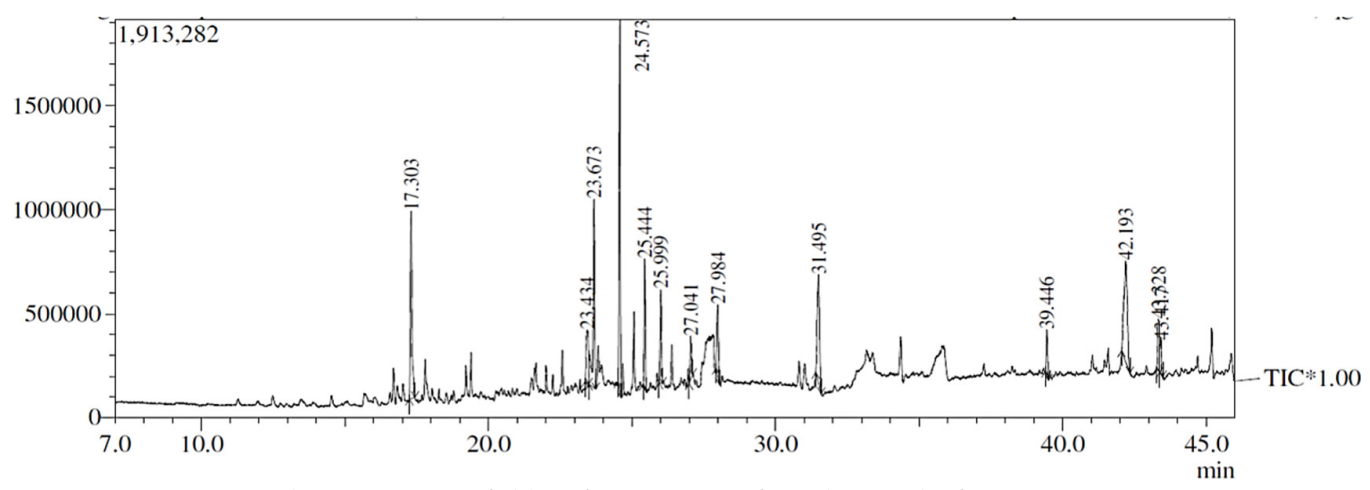

Figure 3. GC-MS chromatogram of chloroform extract of $A$. chinense leaf

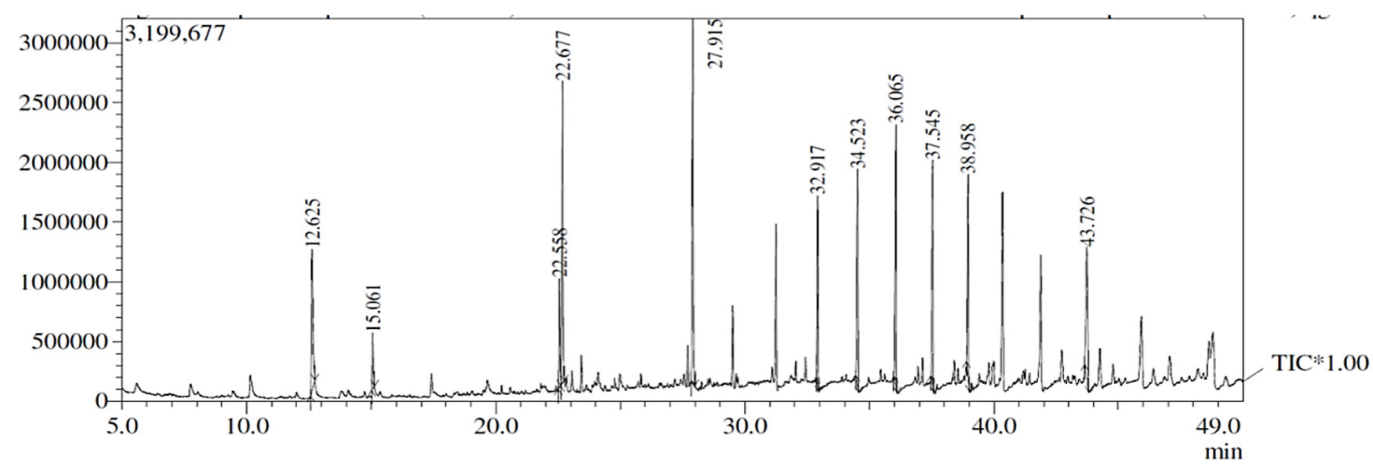

Figure 4. GC-MS chromatogram of petroleum ether extract of $A$. chinense leaf 
Rhetso T et al. (2021). Not Sci Biol 13(2):10791

Table 3. Phytocompounds identified in the ethanol leaf extract of $A$. chinense by GC-MS analysis

\begin{tabular}{|c|c|c|c|c|c|c|}
\hline RT & $\begin{array}{l}\text { Name of the } \\
\text { compound }\end{array}$ & $\begin{array}{c}\text { Peak } \\
\text { area } \%\end{array}$ & $\begin{array}{l}\text { Mol. } \\
\text { formula }\end{array}$ & $\begin{array}{c}\text { Mol. } \\
\text { wt. }\end{array}$ & Structure & Nature \\
\hline 15.991 & $\begin{array}{c}\text { Ethyl } \\
\text { pentadecanoate }\end{array}$ & 1.11 & $\mathrm{C}_{17} \mathrm{H}_{34} \mathrm{O}_{2}$ & 270 & & Fatty acid esters \\
\hline 16.607 & Phytol acetate & 3.95 & $\mathrm{C}_{22} \mathrm{H}_{42} \mathrm{O}_{2}$ & 338 & & Diterpene alcohol \\
\hline 19.010 & $\begin{array}{c}\text { Ethyl 9- } \\
\text { hexadecenoate }\end{array}$ & 1.79 & $\mathrm{C}_{18} \mathrm{H}_{34} \mathrm{O}_{2}$ & 282 & & Fatty acid esters \\
\hline 19.512 & Ethyl palmitate & 18.50 & $\mathrm{C}_{18} \mathrm{H}_{36} \mathrm{O}_{2}$ & 284 & & Fatty acid ethyl ester \\
\hline 22.357 & Phytol & 15.94 & $\mathrm{C}_{20} \mathrm{H}_{40} \mathrm{O}$ & 296 & & Diterpene \\
\hline 23.436 & $\begin{array}{c}\text { Z,Z-6,13- } \\
\text { octadecadien-1-ol } \\
\text { acetate }\end{array}$ & 11.97 & $\mathrm{C}_{20} \mathrm{H}_{36} \mathrm{O}_{2}$ & 308 & & Fatty alcohol \\
\hline 23.613 & $\begin{array}{c}\text { cis,cis,cis-7,10,13- } \\
\text { Hexadecatrienal }\end{array}$ & 15.34 & $\mathrm{C}_{16} \mathrm{H}_{26} \mathrm{O}$ & 234 & & Aldehydes \\
\hline 24.151 & $\begin{array}{c}\text { Ethyl } \\
\text { nonadecanoate }\end{array}$ & 1.23 & $\mathrm{C}_{21} \mathrm{H}_{42} \mathrm{O}_{2}$ & 326 & & Ester \\
\hline 33.261 & 1-Hexacosanol & 3.61 & $\mathrm{C}_{26} \mathrm{H}_{54} \mathrm{O}$ & 382 & M & Primary fatty alcohol \\
\hline 36.231 & Hexatriacontane & 4.57 & $\mathrm{C}_{36} \mathrm{H}_{74}$ & 506 & WM & Alkane \\
\hline 38.638 & $\begin{array}{l}\text { gamma.- } \\
\text { Tocopherol }\end{array}$ & 1.00 & $\mathrm{C}_{28} \mathrm{H}_{48} \mathrm{O}_{2}$ & 416 & & Vitamin E \\
\hline 39.663 & Octacosane & 8.69 & $\mathrm{C}_{28} \mathrm{H}_{58}$ & 394 & & Fatty acid \\
\hline 40.196 & $\begin{array}{l}\text { dl-.alpha.- } \\
\text { Tocopherol }\end{array}$ & 4.94 & $\mathrm{C}_{29} \mathrm{H}_{50} \mathrm{O}_{2}$ & 430 & & Vitamin E \\
\hline 40.726 & Neophytadiene & 3.89 & $\mathrm{C}_{20} \mathrm{H}_{38}$ & 278 & & Sesquiterpenoids \\
\hline 44.565 & Tetratetracontane & 3.47 & $\mathrm{C}_{44} \mathrm{H}_{90}$ & 619 & WMWMMMMUM & Alkane \\
\hline
\end{tabular}

Table 4. Phytocompounds identified in the chloroform leaf extract of $A$. chinense by GC-MS analysis

\begin{tabular}{|c|c|c|c|c|c|c|}
\hline RT & $\begin{array}{c}\text { Name of the } \\
\text { compound }\end{array}$ & $\begin{array}{c}\text { Peak } \\
\text { area } \%\end{array}$ & $\begin{array}{c}\text { Mol. } \\
\text { formula }\end{array}$ & $\begin{array}{c}\text { Mol. } \\
\text { wt. }\end{array}$ & Structure & Nature \\
\hline 27.303 & $\begin{array}{c}2,4-\text { Ditert- } \\
\text { butylphenol }\end{array}$ & 13.80 & $\mathrm{C}_{14} \mathrm{H}_{22} \mathrm{O}$ & 206 & Phenols \\
\hline 23.434 & Calendin & 5.80 & $\mathrm{C}_{11} \mathrm{H}_{16} \mathrm{O}_{3}$ & 196 & & Tetraterpenoids \\
\hline 24.673 & 1 -Nonadecene & 12.09 & $\mathrm{C}_{19} \mathrm{H}_{38} \mathrm{O}$ & 266 & Alkene \\
\hline 24.573 & Phytol acetate & 17.30 & $\mathrm{C}_{22} \mathrm{H}_{42} \mathrm{O}_{2}$ & 338 & & Diterpene alcohol \\
\hline
\end{tabular}


Rhetso T et al. (2021). Not Sci Biol 13(2):10791

\begin{tabular}{|c|c|c|c|c|c|c|}
\hline 25.444 & $\begin{array}{c}3,7,11.15- \\
\text { Tetramethyl-2- } \\
\text { hexadecen-1-ol }\end{array}$ & 6.16 & $\mathrm{C}_{20} \mathrm{H}_{40} \mathrm{O}$ & 296 & & Diterpene \\
\hline 25.999 & $\begin{array}{l}\text { 7,9-Ditert-butyl-1- } \\
\text { oxaspiro[4.5]deca- } \\
\text { 6,9-diene-2,8- } \\
\text { dione }\end{array}$ & 4.74 & $\mathrm{C}_{17} \mathrm{H}_{24} \mathrm{O}_{3}$ & & & Oxaspiro \\
\hline 27.041 & Dibutyl phthalate & 2.56 & $\mathrm{C}_{16} \mathrm{H}_{22} \mathrm{O}_{4}$ & 278 & & Plastilizer \\
\hline 27.984 & 5-Octadecene, (E) & 4.29 & $\mathrm{C}_{18} \mathrm{H}_{36}$ & 252 & & Hydrocarbon alkene \\
\hline 31.495 & Phytol & 10.06 & $\mathrm{C}_{20} \mathrm{H}_{40} \mathrm{O}$ & 296 & & Diterpenoid \\
\hline 39.446 & 1-Hexacosanol & 2.59 & $\mathrm{C}_{26} \mathrm{H}_{54} \mathrm{O}$ & 382 & W & Primary fatty alcohol \\
\hline 42.193 & $\begin{array}{l}\text { Oxalic acid, allyl } \\
\text { tridecyl ester }\end{array}$ & 15.14 & $\mathrm{C}_{18} \mathrm{H}_{32} \mathrm{O}_{4}$ & 312 & & Ester \\
\hline 43.328 & 5-Eicosene, (E) & 3.41 & $\mathrm{C}_{20} \mathrm{H}_{40}$ & 280 & & Alkene \\
\hline 43.417 & Tetradecane & 2.05 & $\mathrm{C}_{14} \mathrm{H}_{30}$ & 198 & & Alkane \\
\hline
\end{tabular}

Table 5. Phytocompounds identified in the petroleum ether leaf extract of $A$. chinense by GC-MS analysis

\begin{tabular}{|c|c|c|c|c|c|c|}
\hline RT & $\begin{array}{l}\text { Name of the } \\
\text { Compound }\end{array}$ & $\begin{array}{c}\text { Peak } \\
\text { area } \%\end{array}$ & $\begin{array}{l}\text { Mol. } \\
\text { formula }\end{array}$ & $\begin{array}{c}\text { Mol } \\
\cdot \\
\text { Wt. }\end{array}$ & Mol. structure & Nature \\
\hline 12.625 & Heptadecane & 8.17 & $\mathrm{C}_{17} \mathrm{H}_{36}$ & 240 & & Alkane \\
\hline 15.061 & Pentadecane & 2.63 & $\mathrm{C}_{15} \mathrm{H}_{32}$ & 212 & 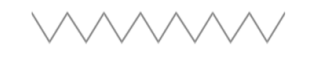 & Alkane \\
\hline 22.558 & Phytol acetate & 3.97 & $\mathrm{C}_{22} \mathrm{H}_{42} \mathrm{O}_{2}$ & 338 & & Diterpene alcohol \\
\hline 22.677 & $\begin{array}{c}\text { Hexahydrofarnesylac } \\
\text { etone }\end{array}$ & 11.79 & $\mathrm{C}_{18} \mathrm{H}_{36} \mathrm{O}$ & 268 & & Ketone \\
\hline 27.915 & Phytol & 15.53 & $\mathrm{C}_{20} \mathrm{H}_{40} \mathrm{O}$ & 296 & & Diterpene \\
\hline 32.917 & Eicosane & 7.52 & $\mathrm{C}_{20} \mathrm{H}_{42}$ & 282 & & Acyclic alkanes \\
\hline 34.523 & Hexadecane & 9.53 & $\mathrm{C}_{16} \mathrm{H}_{34}$ & 226 & & $\begin{array}{c}\text { Alkane } \\
\text { hydrocarbon }\end{array}$ \\
\hline 36.065 & Heptadecane & 11.49 & $\mathrm{C}_{17} \mathrm{H}_{36}$ & 240 & $\wedge$ & Alkane \\
\hline 37.545 & Eicosane & 10.99 & $\mathrm{C}_{20} \mathrm{H}_{42}$ & 282 & MwMm & Acyclic alkanes \\
\hline 38.958 & Octacosane & 8.27 & $\mathrm{C}_{28} \mathrm{H}_{58}$ & 394 & MWMM & Fatty acid \\
\hline 43.726 & Tetracosane & 10.11 & $\mathrm{C}_{24} \mathrm{H}_{50}$ & 338 & WMMMMM & Alkane \\
\hline
\end{tabular}


The most versatile compounds in ethanol leaf extract were ethyl palmitate (18.50\%) which is a longchain fatty acid ethyl ester with has nematicide, antioxidant, anti-androgenic, hemolytic, flavor, and hypocholesterolemic (Tyagi and Agarwal, 2017) activity followed by phytol (15.94\%) a diterpene mostly used as a fragrance in the pharmacological and biotechnological industry has autophagy, antioxidant, anxiolytic, immune-modulating, anti-inflammatory, cytotoxic, metabolism-modulating, apoptosis-inducing, antinociceptive, antimicrobial effects nematicidal, antibacterial, anti-inflammatory and pesticidal activities (Islam et al., 2018; Adnan et al., 2019). No activity has been reported in cis, cis, cis-7,10,13-hexadecatrienal (15.34\%), and Z, Z-6,13-octadecadien-1-ol acetate (11.97\%).

The major compounds found in chloroform leaf extract were phytol acetate (17.30\%) which is acyclic diterpene alcohol has anti-inflammatory, anti-leishmanial activity (Godara et al., 2019). Oxalic acid, allyl tridecyl ester (15.14\%), an ester compound, 2,4-ditert-butylphenol (13.80\%) a phenol has antifungal, antioxidant (Varsha et al., 2015), 1-nonadecene (12.09\%) an unbranched nineteen-carbon alkene has antimicrobial, antioxidant property (Nandhini et al., 2015).

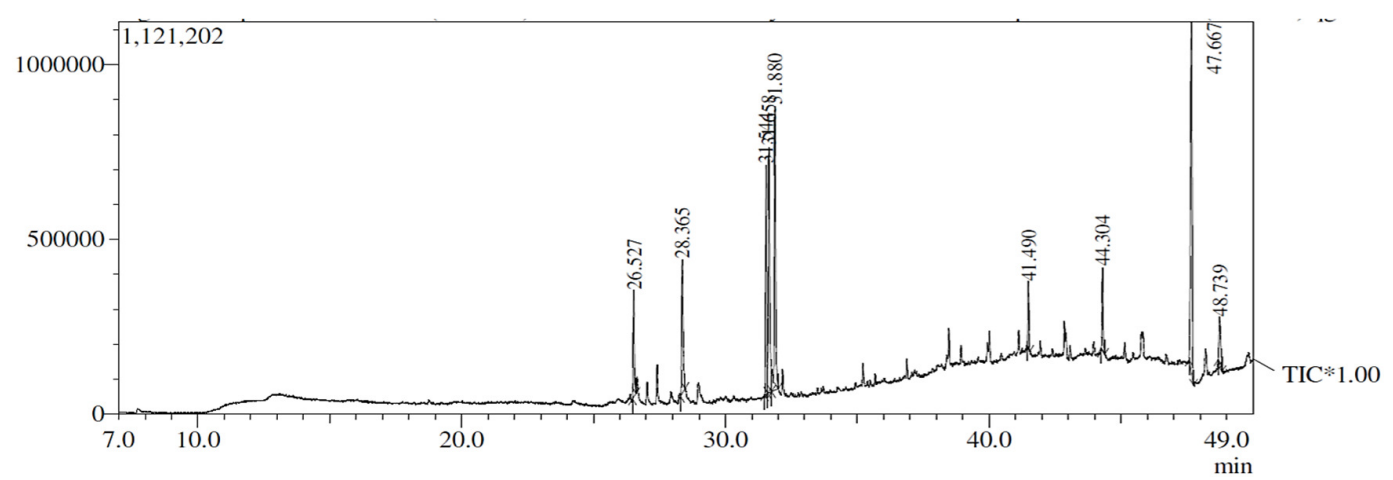

Figure 5. GC-MS chromatogram of methanol extract of $A$. chinense leaf

Table 6. Phytocompounds identified in the methanol leaf extract of $A$. chinense by GC-MS analysis

\begin{tabular}{|c|c|c|c|c|c|c|}
\hline RT & $\begin{array}{l}\text { Name of the } \\
\text { compounds }\end{array}$ & $\begin{array}{c}\text { Peak } \\
\text { area \% }\end{array}$ & $\begin{array}{c}\text { Mol. } \\
\text { formula }\end{array}$ & $\begin{array}{l}\text { Mol. } \\
\text { Wt. }\end{array}$ & Structure & Nature \\
\hline 26.527 & Phytol acetate & 5.58 & $\mathrm{C}_{22} \mathrm{H}_{42} \mathrm{O}_{2}$ & 338 & & Diterpene alcohol \\
\hline 28.365 & $\begin{array}{c}\text { Methyl } \\
\text { isohexadecanoate }\end{array}$ & 9.70 & $\mathrm{C}_{17} \mathrm{H}_{34} \mathrm{O}_{2}$ & 270 & & $\begin{array}{c}\text { Fatty acid methyl } \\
\text { ester }\end{array}$ \\
\hline 31.544 & $\begin{array}{c}\text { Methyl 9,12- } \\
\text { octadecadienoate }\end{array}$ & 12.79 & $\mathrm{C}_{19} \mathrm{H}_{34} \mathrm{O}_{2}$ & 294 & & $\begin{array}{c}\text { Fatty acid methyl } \\
\text { ester }\end{array}$ \\
\hline 31.658 & $\begin{array}{l}\text { Linolenic acid, } \\
\text { methyl ester }\end{array}$ & 16.14 & $\mathrm{C}_{19} \mathrm{H}_{32} \mathrm{O}_{2}$ & 292 & & Linoleic acid \\
\hline 31.880 & Phytol & 17.68 & $\mathrm{C}_{20} \mathrm{H}_{40} \mathrm{O}$ & 296 & & Diterpene \\
\hline 41.490 & Pentatriacontane & 3.10 & $\mathrm{C}_{35} \mathrm{H}_{72}$ & 492 & WWWWWMWW & Alkane \\
\hline 44.304 & Tetratetracontane & 4.45 & $\mathrm{C}_{44} \mathrm{H}_{90}$ & 619 & WWWWWWWWWW & Alkane \\
\hline 47.667 & Octacosane & 27.11 & $\mathrm{C}_{28} \mathrm{H}_{58}$ & 394 & & Alkane \\
\hline 48.739 & Neophytadiene & 3.45 & $\mathrm{C}_{20} \mathrm{H}_{38}$ & 278 & & Sesquiterpenoids \\
\hline
\end{tabular}


It was observed that in the petroleum ether leaf extract the most predominant compounds identified were heptadecane $(19.66 \%)$ a straight-chain alkane has anti-inflammatory, antioxidant, anti-fungal (Kim et al., 2013; Abubacker et al., 2015), eicosane (18.51\%) an acyclic alkane has antioxidant, antibacterial, antifungal (Lin et al., 2016; Chuah et al., 2018), hexahydrofarnesylacetone (11.79\%) a ketone is a pheromone found in numerous plants, frequently as a part of the floral odor, and in some insect species (Schulz et al., 2011) and tetracosane $(10.11 \%)$ a straight-chain alkane that has anti-cancer, cytotoxic property (Paudel et al., 2019).

The most prevailing compounds were octacosane $(27.11 \%)$ a straight-chain alkane that has antioxidant and anti-inflammatory activity Bakr et al., 2017) and phytol (17.68\%) an acyclic diterpene which is also identified in ethanol (15.94\%) and chloroform (10.06\%) extracts were reported to have anti-diuretic, antimicrobial, anticancer, anti-diabetic, anti-inflammatory, and immunostimulatory (U.S. Department of Agriculture, Agricultural Research Service, 1992-2016, Dr. Duke Phytochemical and Ethnobotanical databases,). The compound linolenic acid, methyl ester (16.14\%) has properties like anticandidal and antibacterial activity (Mujeeb et al., 2014) and methyl 9,12-octadecadienoate (12.79\%) has antihistaminic, antieczemic, hepatoprotective, hypocholesterolemic, nematicide, antiandrogenic, antiacne, $5 \alpha$ reductase inhibitor, anticoronary, insectifuge, and antiarthritic (Gnanavel and Saral, 2013; Ghazali and Abdullah, 2014).

All the four extracts of the $A$. chinense leaf exerted a very high amount of saponin content. The ethanol extract had the highest concentration of saponins, flavonoid, and phenol. The GC-MS report showed a high content of terpenoids and alkanes. The metabolite profile reported from the different solvent extracts of the present study varies from the essential oil extracted from both $A$. chinense leaves and bulbs (Pino et al., 2001; Liu et al., 2014). The difference in results may be due to the extraction method, climatic conditions, soil pH, seasonal variation and many other environmental factors.

\section{Conclusions}

To the best of our knowledge, this is the first GC-MS report from India on the metabolite profile of $A$. chinense leaf extracts using methanol, ethanol, petroleum ether, and chloroform solvent. The extracts of $A$. chinense are rich in saponins, terpenes and alkanes representing an important step to understand the phytochemicals constituent of the leaf extracts which could facilitate further use in the pharmaceutical and food industry considering its availability since it is non-toxic and edible. The present results may recommend the use of $A$. chinense leaf to treat various ailments where this plant may be a natural source of a new drug to the scientific and biomedical communities.

\section{Authors' Contributions}

TR; Performed all the experiments and drafted the manuscript. RMS; Participated in carrying out the analysis. SR; Participated in interpretation of the data. SV; Participated in design and critical revision of the manuscript. All authors read and approved the final manuscript.

\section{Acknowledgements}

The first author gratefully acknowledges the University Grant Commission (UGC) of the Government of India for providing Maulana Azad National Fellowship-Senior Research Fellowship (MANF-SRF, F117.1/2017-18-NAG-88047/SA-III/website). The authors express their gratitude to the Department of Botany, Bangalore University, Bangalore -560056 , Karnataka, India for providing facilities to carry out the 
studies and the Central Instrumentation Unit, Kerala Forest Research Institute, Peechi, Thrissur, Kerala, India for GC-MS Analysis of the extracts.

\section{Conflict of Interests}

The authors declare that there are no conflicts of interest related to this article.

\section{References}

Abubacker MN, Devi PK (2015). In vitro antifungal potentials of bioactive compounds heptadecane, 9- hexyl and ethyl iso-allocholate isolated from Lepidagathis cristata Willd. (Acanthaceae) leaf. British Biomedical Bulletin 3(3):336-343.

Adnan M, Chy NU, Mostafa Kamal ATM, Azad MOK, Paul A, Uddin SB, ... Cho DH (2019). Investigation of the biological activities and characterization of bioactive constituents of Ophiorthiza rugosa var. prostrata (D. Don) \& Mondal leaves through in vivo, in vitro, and in silico approaches. Molecules 24(7):1367. https://doi.org/10.3390/molecules24071367

Aryal S, Baniya MK, Danekhu K, Kunwar P, Gurung R and Koirala N (2019). Total phenolic content, flavonoid content and antioxidant potential of wild vegetables from western Nepal. Plants 8(4):96. https://doi.org/10.3390/plants8040096

Bakr RO, El-Naa MM, Zaghloul SS, Omar MM (2017). Profile of bioactive compounds in Nymphaea alba L. leaves growing in Egypt: Hepatoprotective, antioxidant and anti-inflammatory activity. BMC Complementary Medicine and Therapies 17:52. https://doi.org/10.1186/s12906-017-1561-2

Chu C, Cui T, Li S Zhan R, Gao Y (2018). Structure and activity of a new sapogenin from Chlorophytum laxum R. Br. Chemical Research in Chinese Universities 34(5):732-735. https://doi.org/10.1007/s40242-018-8114-1

Chuah XQ, Okechukwu PN, Amini F, Teo SS (2018). Eicosane, pentadecane and palmitic acid: The effects in in vitrowound healing studies. Asian Pacific Journal of Tropical Medicine 8(10):490-499. https://doi.org/10.4103/2221-1691.244158

Ghazali N, Abdullah NA (2014). GC-MS analysis of some bioactive components in the root extract of Linn Ixora coccinea Linn. International Journal of Pharma and Bio Sciences 5(3):197-203.

Gnanavel V, Saral MA (2013). GC-MS analysis of petroleum ether and ethanol leaf extracts from Abrus precatorius Linn. International Journal of Pharm and Bio Sciences 4(3):37-44.

Godara P, Dulara BK, Barwer NB, Chaudhary NS (2019). Comparative GC-MS analysis of bioactive phytochemicals from different plant parts and callus of Leptadenia reticulata Wight and Arn. Pharmacognosy Journal 11(1):129-140 http://dx.doi.org/10.5530/pj.2019.1.22

Hosoki T, Hiura H, Hamada M (1985). Breaking bud dormancy in corms, tubers, and trees with sulfur-containing compounds HortScience 20: 290-291.

Hussain G, Rasul A, Anwar H, Aziz N, Razzaq A, Wei Wei, ... Li X (2018). Role of plant derived alkaloids and their mechanism in neurodegenerative disorders. International Journal of Biological Sciences 14(3):341-357 https://doi.org/10.7150/ijbs.23247

Islam MT, Ali ES, Uddin SJ, Shaw S, Islam MA, Ahmed MI, ... Atanasov AG (2018). Phytol: A review of biomedical activities. Food and Chemical Toxicology 121:82-94. https://doi.org/10.1016/j.fct.2018.08.032

Khan AN, Bhat I (2018). Extraction, qualitative and quantitative determination of secondary metabolites of Rumex nepalensis Roots. Journal of Drug Delivery and Therapeutics 8(6):97-100. https://doi.org/10.22270/jddt.v8i6s. 2092

Kim DH, Park MH, Choi YJ, Chung KW, Park CH, Jang EJ ... Chung HY (2013). Molecular study of dietary heptadecane for the anti-inflammatory modulation of NF-kB in the aged kidney. PLoS One 8(3):1-10. https://doi.org/10.1371/journal.pone.0059316

Kretovich UL (2005). Principles of plant biochemistry. Permagon, Oxford Press. Journal of Food Science 54:254-260. 
Kubota N, Matthews MA, Takahagi T, Kliewer WM (2000). Budbreak with garlic preparations: Effects of garlic preparations and of calcium and hydrogen cyanamides on budbreak of grapevines grown in greenhouses. American Journal of Enology and Viticulture 51:409-414.

Le AV, Parks SE, Nguyen MH, Roach PD (2018). Improving the vanillin-sulphuric acid method for quantifying total saponins. Technologies 6(3):84. https://doi.org/10.3390/technologies6030084

Lefahal M, Zaabat N, Ayad R, Makhloufi EH, Djarri L, Benahmed M, ... Akkal S (2018). In vitro assessment of total phenolic and flavonoid contents, antioxidant and photoprotective activities of crude methanolic extract of aerial parts of Capnophyllum Peregrinum(L.) Lange (Apiaceae) growing in Algeria. Medicines 5(2):26. https://doi.org/10.3390/medicines5020026

Lim TK (2015). Edible medicinal and non-medicinal plants, Allium chinense. 9 Modified Stems, Roots, Bulbs Springer, Dordrecht pp 204-209.

Lin YP, Lin LY, Yeh HY, Chuang CH, Tseng SW, Yen YH (2016). Antihyperlipidemic activity of Allium chinense bulbs. Journal of Food and Drug Analysis 24(3):516-526. https://doi.org/10.1016/j.jfda.2016.01.010

Liu XC, Lu XN, Liu QZ, Liu ZL (2014). Evaluation of insecticidal activity of the essential oil of Allium chinense G. Don and its major constituents against Liposcelis bostrychophila Badonnel. Journal of Asia-Pacific Entomology 17(4):853-856. https://doi.org/10.1016/j.aspen.2014.08.007

Liu Y, Wang M, Liu K, Qiu P, Zhang S, Lu Y, ... Tang H (2018). New steroidal saponins from the rhizomes of Paris vietnamensis and their cytotoxicity. Molecules 23(3):588. https://doi.org/10.3390/molecules23030588

Lobo V, Patil A, Phatak A, Chandra N (2010). Free radicals, antioxidants and functional foods: Impact on human health. Pharmacognosy Reviews 4(8):118-126. https://dx.doi.org/10.4103\%2F0973-7847.70902

Merculieff1 Z, Ramnath S, Sankoli SM, Venkataramegowda S, Murthy, GS, Ceballos RM (2014). Phytochemical, antioxidant and antibacterial potential of Elaeagnus kologa (Schlecht.) leaf. Asian Pacific Journal of Tropical Medicine 4(9):687-691. https://doi.org/10.1016/S1995-7645(14)60295-9

Mujeeb F, Bajpai P, Pathak N (2014). Phytochemical evaluation, antimicrobial activity and determination of bioactive components from leaves of Aegle marmelos. Biomed Research International https://doi.org/10.1155/2014/497606

Nandhini US, Sangareshwari S, Lata K (2015). Gas chromatography-mass spectrometry analysis of bioactive constituents from the marine Streptomyces. Asian Journal of Pharmaceutical and Clinical Research 8(2):244-246.

Paudel MR, Chand MB, Pant B, Pant B (2019). Assessment of antioxidant and cytotoxic activities of extracts of Dendrobium crepidatum. Biomolecules 9(9):478. https://doi.org/10.3390/biom9090478

Pereira DM, Valentao P, Pereira JA, Andrade PB (2009). Phenolics: from chemistry to biology. Molecules 14(6):22022211 https://doi.org/10.3390/molecules 14062202

Pino JA, Fuentes V, Correa MT (2001). Volatile constituents of Chinese chive (Allium tuberosum Rottl. ex Sprengel) and rakkyo (Allium chinense G. Don). Journal of Agricultural and Food Chemistry 49(3):1328-1330. https://doi.org/10.1021/jf9907034

Qiu T, Wu D, Yang L, Ye H, Wang Q, Cao Z, Tang K (2018). Exploring the mechanism of flavonoids through systematic bioinformatics analysis. Frontiers in Pharmacology 9:918. https://doi.org/10.3389/fphar.2018.00918

Safari M, Ahmady-Asbchin S (2019): Evaluation of antioxidant and antibacterial activities of methanolic extract of medlar (Mespilus germanicaL.) leaves. Biotechnology and Biotechnological Equipment 33:372-378. https://doi.org/10.1080/13102818.2019.1577701

Schulz S, Yildizhan S, Loon JJAV (2011). The Biosynthesis of hexahydrofarnesylacetone in the butterfly Pieris brassicae. Journal of Chemical Ecology 37(4):360-363. https://doi.org/10.1007/s10886-011-9939-y

Sobolewska D, Galanty A, Grabowska K, Makowska-Was J, Wrobel-Biedrawa D, Podolak I (2020). Saponins as cytotoxic agents: an update (2010-2018). Part I-steroidal saponins. Phytochemistry Reviews 19:139-189. https://doi.org/10.1007/s11101-020-09661-0

Tan PV (2018). The Determination of total alkaloid, polyphenol, flavonoid and saponin contents of Pogang gan (Curcuma sp.). International Journal of Biology 10(4):42-47. https://doi.org/10.5539/ijb.v10n4p42

Thawabteh A, Lelario F, Scrano L, Bufo SA, Nowak S, Behrens M, ... Karaman R (2019). Bitterless guaifenesin prodrugsdesign, synthesis, characterization, in vitro kinetics, and bitterness studies. Chemical Biology and Drug Design 93(3):262-271. https://doi.org/10.1111/cbdd.13409

Tyagi T, Agarwal M (2017). Phytochemical screening and GC-MS analysis of bioactive constituents in the ethanolic extract of Pistia stratiotes L. and Eichhornia crassipes (Mart.) Solms. Journal of Pharmacognosy and Phytochemistry 6(1):195-206. 
U.S. Department of Agriculture, Agricultural Research Service (1992-2016). Dr. Duke's Phytochemical and Ethnobotanical Databases. Retrieved 2016 Mar 23 from http://phytochem.nal.usda.gov/ http://dx.doi.org/10.15482/USDA.ADC/1239279

Varsha KK, Devendra L, Shilpa G, Priya S, Pandey A, Nampoothiri KM (2015).2,4-Di-tert-butyl phenol as the antifungal, antioxidant bioactive purified from a newly isolated Lactococcus sp. International Journal of Food Microbiology 211:44-50. https://doi.org/10.1016/j.ijfoodmicro.2015.06.025

Vasundhara M, Nethravathi M, Priyanka R, Marappa N, Gujaran SR (2017). Antioxidant potential of Laurus nobilis L. essential oil. Agricultural Research Journal 54(4):495-499.

http://dx.doi.org/10.5958/2395-146X.2017.00095.3

Wang B, Ma MP, Diao QY, Tu Y (2019). Saponin-induced shifts in the rumen microbiome and metabolome of young cattle. Frontiers in Microbiology. https://doi.org/10.3389/fmicb.2019.00356

Wang J, Song H, Wu X, Zhang S, Gao X, Li F, ... Chen Q (2018). Steroidal saponins from Vernonia amygdalina Del. and their biological activity. Molecules 23(3):579. https://doi.org/10.3390/molecules23030579

Wang TY, Li Q, Bi KS (2017). Bioactive flavonoids in medicinal plants: Structure, activity and biological fate. Asian Journal of Pharmaceutical Sciences 13:12-23. https://doi.org/10.1016/j.ajps.2017.08.004

Yang B, Xu B, Zhao H, Wang YB, Zhang J, Li CW, ... Cao F (2018). Dioscin protects against coronary heart disease by reducing oxidative stress and inflammation via Sirt1/Nrf2 and p38 MAPK pathways. Molecular Medicine Reports 18(1):973-980. https://doi.org/10.3892/mmr.2018.9024

Yao ZH, Qin ZF, Dai Y, Yao XS (2016). Phytochemistry and pharmacology of Allii macrostemonis Bulbus, a traditional Chinese medicine. Chinese Journal of Natural Medicines 14(7):481-498.

https://doi.org/10.1016/s1875-5364(16)30058-9
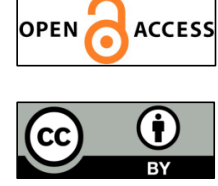

The journal offers free, immediate, and unrestricted access to peer-reviewed research and scholarly work. Users are allowed to read, download, copy, distribute, print, search, or link to the full texts of the articles, or use them for any other lawful purpose, without asking prior permission from the publisher or the author.

License - Articles published in Notulae Scientia Biologicae are Open-Access, distributed under the terms and conditions of the Creative Commons Attribution (CC BY 4.0) License.

(c) Articles by the authors; SHST, Cluj-Napoca, Romania. The journal allows the author(s) to hold the copyright/to retain publishing rights without restriction. 